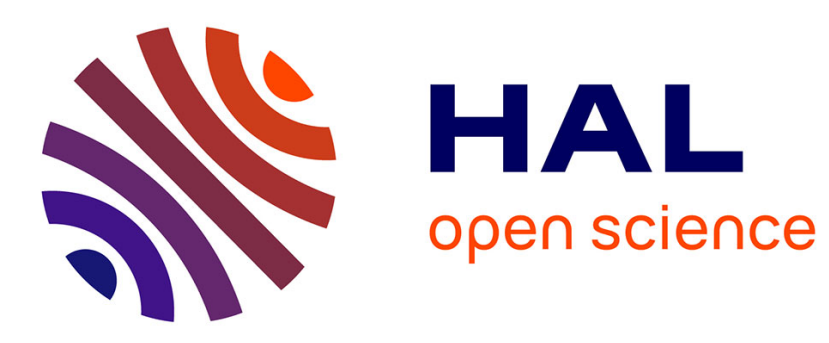

\title{
A Completion Method to Decide Reachability in Rewrite Systems
}

Guillaume Burel, Gilles Dowek, Ying Jiang

\section{To cite this version:}

Guillaume Burel, Gilles Dowek, Ying Jiang. A Completion Method to Decide Reachability in Rewrite Systems. International Symposium on Frontiers of Combining Systems FroCoS'15, Sep 2015, Wroclaw, Poland. pp.205-219, 10.1007/978-3-319-24246-0_13 . hal-01252138

\section{HAL Id: hal-01252138 https://hal.inria.fr/hal-01252138}

Submitted on 7 Jan 2016

HAL is a multi-disciplinary open access archive for the deposit and dissemination of scientific research documents, whether they are published or not. The documents may come from teaching and research institutions in France or abroad, or from public or private research centers.
L'archive ouverte pluridisciplinaire HAL, est destinée au dépôt et à la diffusion de documents scientifiques de niveau recherche, publiés ou non, émanant des établissements d'enseignement et de recherche français ou étrangers, des laboratoires publics ou privés. 


\title{
A completion method to decide reachability in rewrite systems
}

\author{
Guillaume Burel ${ }^{1}$, Gilles Dowek ${ }^{2}$ and Ying Jiang ${ }^{3}$ \\ ${ }^{1}$ Ensiie, 1 square de la Résistance, 91000 Évry, France. \\ ${ }^{2}$ Inria, 23 avenue d'Italie, CS 81321, 75214 Paris Cedex 13, France. \\ ${ }^{3}$ State Key Laboratory of Computer Science, Institute of Software, Chinese Academy of \\ Sciences, 100190 Beijing, China. \\ Email: jy@ios.ac.cn
}

\begin{abstract}
The Knuth-Bendix method takes in argument a finite set of equations and rewrite rules and, when it succeeds, returns an algorithm to decide if a term is equivalent to another modulo these equations and rules. In this paper, we design a similar method that takes in argument a finite set of rewrite rules and, when it succeeds, returns an algorithm to decide not equivalence but reachability modulo these rules, that is if a term reduces to another. As an application, we give new proofs of the decidability of reachability in finite ground rewrite systems and in pushdown systems.
\end{abstract}

\section{INTRODUCTION}

The Knuth-Bendix method [13, 10] takes in argument a finite set of equations and rewrite rules and, when it succeeds, returns an algorithm to decide if a term is equivalent to another modulo these equations and rules. In this paper, we design a similar method that takes in argument a finite set of rewrite rules and, when it succeeds, returns an algorithm to decide not equivalence but reachability modulo these rules, that is if a term reduces to another.

As an application, we give new proofs of the decidability of reachability in finite ground rewrite systems [3] and in pushdown systems [1].

Like the Knuth-Bendix method, this method proceeds by completing a finite rewrite system into an equivalent one, by adding derivable rules. In the completed system, when a proposition $t \longrightarrow^{*} u$ has a proof, it also has a proof of the form

$$
t=t_{0} \longrightarrow t_{1} \ldots \longrightarrow t_{p}=w=u_{q} \longrightarrow \ldots u_{1} \longrightarrow u_{0}=u
$$

where $t_{0} \succ t_{1} \succ \ldots \succ t_{p}$ and $u_{q} \prec \ldots \prec u_{1} \prec u_{0}$ for some reduction order $\prec$, that is a proof formed with a decreasing sequence followed by an increasing one. We can write such a proof

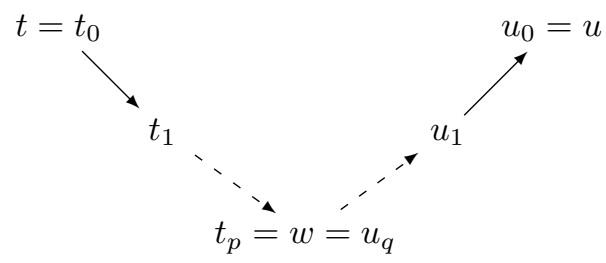

using the unusual convention to write the larger terms for the order $\prec$ on the top of the diagram and the smaller ones on the bottom, hence drawing an arrow oriented from the bottom to the top, when a smaller term reduces to a larger one.

In order to transform proofs into proofs of this form, we should consider critical steps of the form

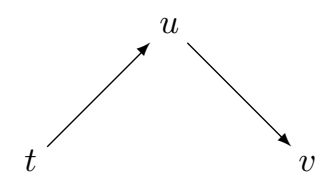

with $t \prec u \succ v$ and add a rule reducing directly $t$ to $v$, avoiding the detour via $u$. If the reduction from $t$ to $u$ uses a rule $l_{1} \longrightarrow r_{1}$ and that from $u$ to $v$ a rule $l_{2} \longrightarrow r_{2}$, the terms $r_{1}$ and $l_{2}$ would have to be compared, to determine if one term unifies with a subterm of the other. We would therefore need to design a forward completion method that compares the lefthand side of a rule with the right-hand side of another.

An alternative method is to reverse the rules whose left-hand side is smaller than the right-hand side, and keep track that such reversed rules must be used backwards. Thus, we distinguish two kinds of rules: negative rules that are as usual, and positive rules that must be used backwards: $u_{1} \longrightarrow+u_{2}$ means that $u_{2} \longrightarrow u_{1}$ in the original system and $u_{2} \prec u_{1}$. This way, in the completed system, when a proposition $t \longrightarrow{ }^{*} u$ has a proof, it also has a proof of the form

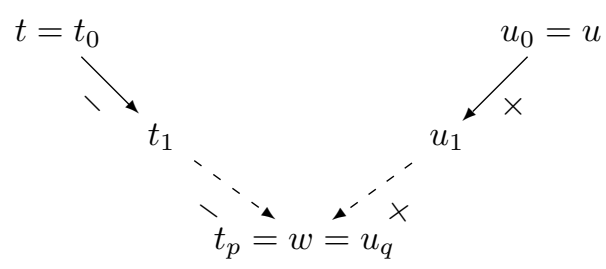


and the critical steps have the form

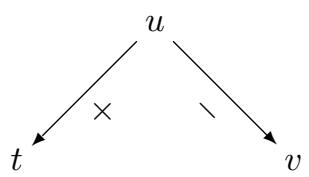

so that only left-hand side of rules need to be compared.

In the completed system, the proposition $t \longrightarrow^{*} u$ has a proof if and only if there exists a term $w$ such that $t \longrightarrow_{-}^{*} w$ and $u \longrightarrow_{+}^{*} w$. Thus reachability boils down to the existence of a common reduct. In a terminating system, reachability is obviously decidable and easy to check because reduction trees are always finite. In the same way, in a terminating system, the existence of a common reduct of two terms is decidable and easy to check because reduction trees are finite.

The reader familiar with polarized sequent calculus modulo theory $[6,8]$ will remark that many ideas in this paper, in particular the idea to distinguish two kinds of rules, come from this calculus. But the paper is presented independently of polarized sequent calculus modulo theory.

\section{POLARIZED REWRITE SYSTEMS}

We consider a finite set constants $a, b, \ldots$ a finite set of function symbols $f, g, \ldots$ and an infinite set of variables, $x, y, X, Y, \ldots$ Terms, substitutions, rewrite rules, and rewrite systems are defined as usual.

A rewrite rule $l \longrightarrow r$ is said to be left-linear if the term $l$ is linear in each of its variables, that is if each variable of $l$ occurs exactly once in $l$.

A context $C\left[X_{1}, \ldots, X_{n}\right]$ is an ordered pair formed with a term $C$, and a sequence of variables $X_{1}, \ldots, X_{n}$ each occurring exactly once in $C$. The term $\left(t_{1} / X_{1}, \ldots, t_{n} / X_{n}\right) C$ is written $C\left[t_{1}, \ldots, t_{n}\right]$.

Definition 2.1 (Polarized rewrite system). A polarized rewrite system $\mathcal{P}$ is a pair $\left\langle\mathcal{P}_{-}, \mathcal{P}_{+}\right\rangle$of rewrite systems. The rules of $\mathcal{P}_{-}$are called negative and are written $l \longrightarrow_{-} r$, the rules of $\mathcal{P}_{+}$are called positive and are written $l \longrightarrow_{+} r$. The one step reduction relations $\longrightarrow_{-}$and $\longrightarrow_{+}$are defined as usual: $t \longrightarrow_{-} u$ (resp. $t \longrightarrow_{+} u$ ) if there exists a negative rule $l \longrightarrow_{-} r$ (resp. a positive rule $l \longrightarrow+r$ ), a context $C[X]$ and a substitution $\sigma$, such that $t=C[\sigma l]$ and $u=C[\sigma r]$.

Definition 2.2 (The relation $\longrightarrow$ ). The relation $\longrightarrow$ is $\longrightarrow_{-} U_{+} \longleftarrow$, that is $t \longrightarrow u$ if $t \longrightarrow_{-} u$ or $u \longrightarrow_{+} t$.

Definition 2.3 (Proof). Let $\mathcal{P}$ be a polarized rewrite system. A proof (or a reduction sequence) in $\mathcal{P}$ is a sequence of terms $t_{0}, t_{1}, \ldots, t_{n}$, such that for all $i$, $t_{i} \longrightarrow t_{i+1}$, that is $t_{i} \longrightarrow_{-} t_{i+1}$ or $t_{i+1} \longrightarrow_{+} t_{i}$.

A proof is a proof of the proposition $t \longrightarrow^{*} u$ if $t=t_{0}$ and $u=t_{n}$.

Definition 2.4 (Polarization). The polarized rewrite system $\mathcal{P}$ is said to be a polarization of a non-polarized rewrite system $\mathcal{R}$ if
- for each rule $l \longrightarrow r$ of $\mathcal{R}$, the system $\mathcal{P}$ contains either the rule $l \longrightarrow_{-} r$ or the rule $r \longrightarrow_{+} l$,

- for each rule $l \longrightarrow$ - $r$ of $\mathcal{P}$, the system $\mathcal{R}$ contains the rule $l \longrightarrow r$,

- and, for each rule $l \longrightarrow_{+} r$ of $\mathcal{P}$, the system $\mathcal{R}$ contains the rule $r \longrightarrow l$.

Proposition 2.1. Let $\mathcal{R}$ be a rewrite system, and $\mathcal{P}$ be a polarization of $\mathcal{R}$, then a proposition $t \longrightarrow^{*} u$ has a proof in $\mathcal{R}$ if and only if it has a proof in $\mathcal{P}$.

EXAMPLE 1 . Let $\mathcal{R}$ be the rewrite system

$$
\begin{aligned}
f(g(a)) & \longrightarrow b \\
f(f(a)) & \longrightarrow c \\
b & \longrightarrow g(g(f(f(a)))) \\
c & \longrightarrow h(a)
\end{aligned}
$$

The polarized rewrite system $\mathcal{P}$

$$
\begin{aligned}
f(g(a)) & \longrightarrow_{-} b \\
f(f(a)) & \longrightarrow_{-} c \\
g(g(f(f(a)))) & \longrightarrow_{+} b \\
h(a) & \longrightarrow_{+} c
\end{aligned}
$$

is a polarization of $\mathcal{R}$. The proposition $f(g(a)) \longrightarrow^{*}$ $g(g(h(a)))$ has the following proof in $\mathcal{R}$

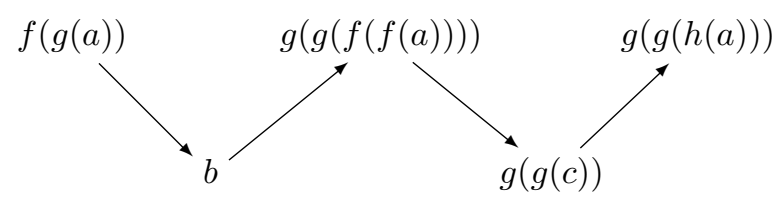

and the following proof in $\mathcal{P}$

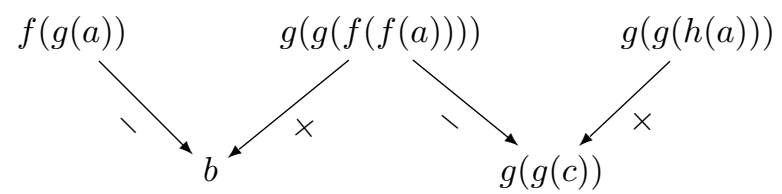

Definition 2.5 (Termination). A polarized rewrite system is terminating if the relation $\longrightarrow_{-} \cup \longrightarrow_{+}$is well-founded.

Note that this does not imply that the relation $\longrightarrow$, that is $\longrightarrow_{-} U_{+} \longleftarrow$, is well-founded.

Definition 2.6 (Reduction order). A reduction order $\prec$ is an order such that

- if $t \prec u$, then for all function symbols $f$ and terms $t_{1}, \ldots, t_{i-1}, t_{i+1}, \ldots, t_{n}$

$$
\begin{gathered}
f\left(t_{1}, \ldots, t_{i-1}, t, t_{i+1}, \ldots, t_{n}\right) \\
\prec \\
f\left(t_{1}, \ldots, t_{i-1}, u, t_{i+1}, \ldots, t_{n}\right)
\end{gathered}
$$

- if $t \prec u$, then for all substitutions $\sigma$

$$
\sigma t \prec \sigma u
$$

- $\prec$ is well-founded.

Proposition 2.2. Let $\prec$ be a reduction order and $\mathcal{P}$ be a polarized rewrite system such that $l \succ r$ for each rule $l \longrightarrow_{-} r$ or $l \longrightarrow_{+} r$ of $\mathcal{P}$. Then, the system $\mathcal{P}$ terminates. 


\section{CUT-ELIMINATION}

Definition 3.1 (Cut). A cut (or a peak) in a proof $\pi=t_{0}, t_{1}, \ldots, t_{n}$ is a sub-sequence $t_{i-1}, t_{i}, t_{i+1}$ such that

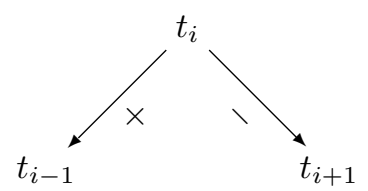

A proof is cut-free (or a valley proof) if it contains no cuts, that is if it is formed with a sequence of negative steps followed by a sequence of positive steps.

$A$ polarized rewrite system has the cut-elimination property (or is confluent) if every proposition $t \longrightarrow^{*} u$ that has a proof has a cut-free proof.

EXAMPLE 2. In the polarized rewrite system $\mathcal{P}$ of Example 1, the proposition $f(g(a)) \longrightarrow^{*} g(g(h(a)))$ has the proof

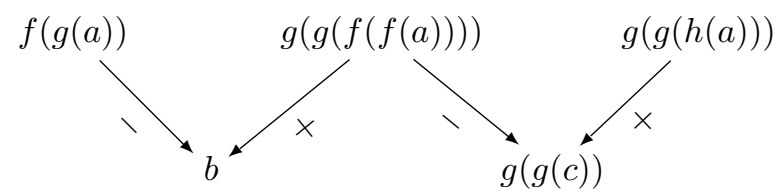

but no cut-free proof.

The reader familiar with polarized sequent calculus modulo theory will remark that the proposition $t \longrightarrow^{*} u$ has a proof (resp. a cut-free proof) in $\mathcal{P}$ if and only if the sequent $P(t) \vdash P(u)$, where $P$ is a predicate symbol, has a proof (resp. a cut-free proof) in polarized sequent calculus modulo $\mathcal{P}$.

Proposition 3.1. Let $\mathcal{P}$ be a terminating finite polarized rewrite system. Then, the existence of a cutfree proof in $\mathcal{P}$ of a proposition $t \longrightarrow^{*} u$ is decidable.

Proof. The proposition $t \longrightarrow{ }^{*} u$ has a cut-free proof if and only if the reducts of $t$ in $\mathcal{P}_{-}$and those of $u$ in $\mathcal{P}_{+}$have a term in common. As $\mathcal{P}$ terminates, both reduction trees are finite.

Definition 3.2 (Proof reduction). A proof $\pi$ reduces to a proof $\pi^{\prime}$, if $\pi^{\prime}$ is obtained by replacing a cut in $\pi$ by a cut-free proof, that is if

$$
\pi=t_{0}, \ldots, t_{i-1}, t_{i}, t_{i+1}, \ldots, t_{n}
$$

with

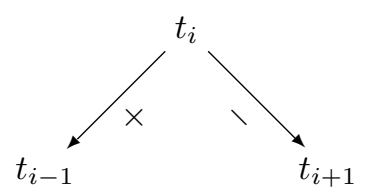

and

$\pi^{\prime}=t_{0}, \ldots, t_{i-1}=u_{0}, u_{1}, \ldots, u_{p}=w=v_{q}, \ldots, v_{1}$, $v_{0}=t_{i+1}, \ldots, t_{n}$ with

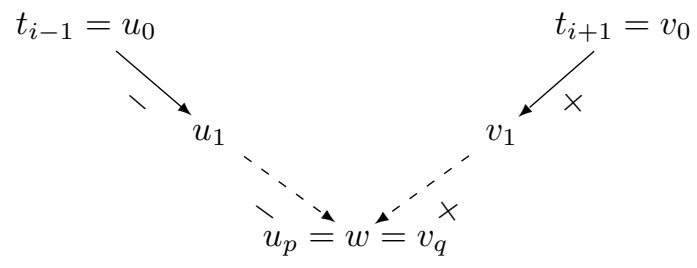

Definition 3.3 (Local confluence). A polarized rewrite system is locally confluent if every cut is reducible, that is if for each proof

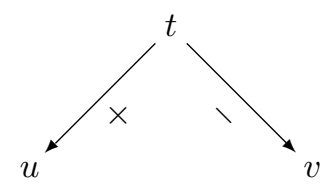

there exists a proof

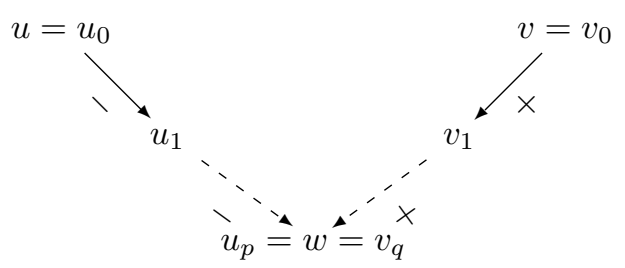

Newman's lemma can be seen as a termination lemma for proof-reduction [7], following an idea that is already in $[11]$.

Proposition 3.2 (Newman). If $\mathcal{P}$ is terminating and locally confluent, then it has the cut-elimination property.

Proof. As $\mathcal{P}$ is terminating, the transitive closure of the relation $\longrightarrow_{-} \cup \longrightarrow_{+}$is a well-founded order. Thus, its multiset extension $<[5]$ is also well-founded. A proof-reduction step replaces the multiset $\left\{t_{1}, \ldots, t_{i-1}, t_{i}, t_{i+1}, \ldots, t_{n}\right\}$ with the multiset $\left\{t_{1}, \ldots, t_{i-1}, u_{1}, \ldots, u_{p-1}, w, v_{q-1}, \ldots, v_{1}, t_{i+1}, \ldots, t_{n}\right\}$ and

$$
\begin{gathered}
\left\{t_{1}, \ldots, t_{i-1}, u_{1}, \ldots, u_{p-1}, w, v_{q-1}, \ldots, v_{1}, t_{i+1}, \ldots, t_{n}\right\} \\
< \\
\left\{t_{1}, \ldots, t_{i-1}, t_{i}, t_{i+1}, \ldots, t_{n}\right\}
\end{gathered}
$$

because each term $u_{1}, \ldots, u_{p-1}, w, v_{q-1}, \ldots, v_{1}$ is smaller than $t_{i}$. Thus, proof-reduction terminates.

Finally, as $\mathcal{P}$ is locally confluent, an irreducible proof contains no cuts.

Definition 3.4 (Critical pair). A critical pair is a pair of terms of the form

- $\left\langle\sigma r_{1},(\sigma C)\left[\sigma r_{2}\right]\right\rangle$, where $l_{1} \longrightarrow_{-} r_{1}$ is a negative rule, $l_{2} \longrightarrow+r_{2}$ is a positive rule, $C[X]$ is a context, $l_{1}^{\prime}$ is a term that is not a variable, and $\sigma$ is a substitution, such that $X$ does not occur in $\sigma, l_{1}=C\left[l_{1}^{\prime}\right]$, and $\sigma$ is the most general unifier of $l_{1}^{\prime}$ and $l_{2}$,

- or $\left\langle(\sigma C)\left[\sigma r_{1}\right], \sigma r_{2}\right\rangle$, where $l_{1} \longrightarrow_{-} r_{1}$ is a negative rule, $l_{2} \longrightarrow+r_{2}$ is a positive rule, $C[X]$ is a context, $l_{2}^{\prime}$ is a term that is not a variable, and 
$\sigma$ is a substitution, such that $X$ does not occur in $\sigma, l_{2}=C\left[l_{2}^{\prime}\right]$, and $\sigma$ is the most general unifier of $l_{1}$ and $l_{2}^{\prime}$.

$A$ critical pair $\langle u, v\rangle$ is joinable if there exists a term $w$, such that the propositions $u \longrightarrow_{+}^{*} w$ and $v \longrightarrow{ }_{-}^{*} w$ are provable.

We now would like to prove that if all the critical pairs of a polarized rewrite system $\mathcal{P}$ are joinable, then $\mathcal{P}$ is locally confluent. Unfortunately, this property does not hold in general, as shown by the following counterexample

$$
\begin{gathered}
f(x, x) \longrightarrow_{-} g(x) \\
a \longrightarrow_{+} b
\end{gathered}
$$

that contains no critical pairs, but that is not locally confluent, as the cut

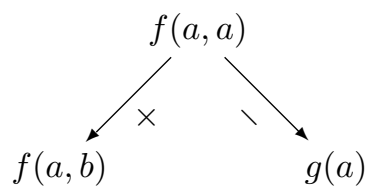

cannot be reduced: the term $g(a)$ reduces positively to $g(b)$ only, and the term $f(a, b)$ cannot be reduced negatively. It indeed reduces to $f(b, b)$, but not negatively. N. Hirokawa [9] has found a similar counter-example independently, in a different context. Fortunately, this property holds for left-linear rewrite systems.

We start by recalling two well-known classification lemmas $[13,10]$.

Proposition 3.3. Let $C_{1}[X]$ and $C_{2}[Y]$ be contexts, and $u_{1}$ and $u_{2}$ be terms such that $C_{1}\left[u_{1}\right]=C_{2}\left[u_{2}\right]$ then

- either the occurrences of $X$ and $Y$ are disjoint, that is there exists a context $D[X, Y]$ such that $C_{1}[X]=D\left[X, u_{2}\right]$ and $C_{2}[Y]=D\left[u_{1}, Y\right]$
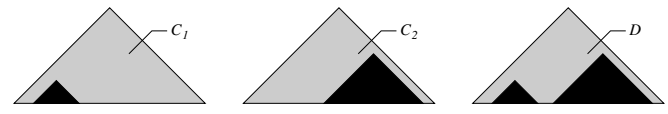

- or the occurrence of $X$ is higher than that of $Y$, that is there exists a context $D[Y]$ such that $C_{2}[Y]=C_{1}[D[Y]]$
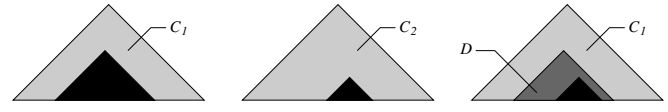

- or the occurrence of $Y$ is higher than that of $X$, that is there exists a context $D[X]$ such that $C_{1}[X]=C_{2}[D[X]]$
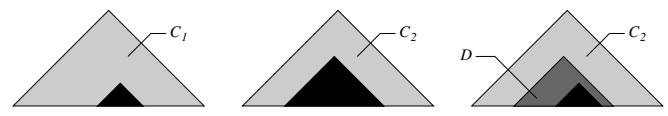

Proposition 3.4. Let $t$ and $u$ be terms, $\tau$ be $a$ substitution and $D[Y]$ be a context such that the variable $Y$ does not occur in $\tau$ and $\tau t=D[u]$. Then
- either the occurrence of $Y$ in $D$ is not an occurrence of $t$, that is there exist a variable $x$ and contexts $E_{1}[X]$ and $E_{2}[Y]$, such that $t=E_{1}[x]$, $\tau x=E_{2}[u]$, and $D[Y]=\left(\tau E_{1}\right)\left[E_{2}[Y]\right]$
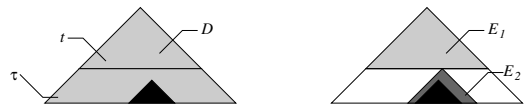

- or the occurrence of $Y$ in $D$ is an occurrence of $t$, that is there exist a context $E$ and a term $t^{\prime}$ such that $t=E\left[t^{\prime}\right]$ and $D[Y]=(\tau E)[Y]$
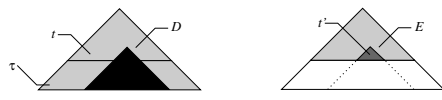

Proposition 3.5. If all the critical pairs of a leftlinear polarized rewrite system $\mathcal{P}$ are joinable, then $\mathcal{P}$ is locally confluent.

Proof. Consider three terms $t, u$, and $v$ such that

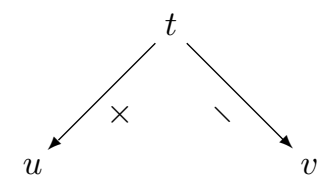

where $t$ reduces to $u$ by a rule $l_{1} \longrightarrow_{+} r_{1}$, and to $v$ by a rule $l_{2} \longrightarrow_{-} r_{2}$. As variables in rules may be renamed we can assume, without loss of generality, that $l_{1}$ and $l_{2}$ have no variables in common.

There exist two contexts $C_{1}[X]$ and $C_{2}[Y]$ and a substitution $\tau$, such that $X$ and $Y$ do not occur in $\tau, t=C_{1}\left[\tau l_{1}\right]=C_{2}\left[\tau l_{2}\right], u=C_{1}\left[\tau r_{1}\right]$, and $v=$ $C_{2}\left[\tau r_{2}\right]$. Thus, by Proposition 3.3, either there exists a context $D[X, Y]$ such that $C_{1}[X]=D\left[X, \tau l_{2}\right]$ and $C_{2}[Y]=D\left[\tau l_{1}, Y\right]$, or there exists a context $D[Y]$ such that $C_{2}[Y]=C_{1}[D[Y]]$, or there exists a context $D[X]$ such that $C_{1}[X]=C_{2}[D[X]]$. We consider these three cases.

- If there exists a context $D[X, Y]$ such that $C_{1}[X]=D\left[X, \tau l_{2}\right]$ and $C_{2}[Y]=D\left[\tau l_{1}, Y\right]$

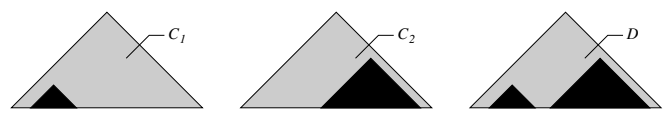

we have $u=D\left[\tau r_{1}, \tau l_{2}\right]$, and $v=D\left[\tau l_{1}, \tau r_{2}\right]$, let $w=D\left[\tau r_{1}, \tau r_{2}\right]$. We have

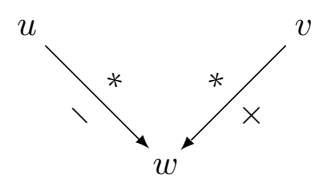

- If there exists a context $D[Y]$ such that $C_{2}[Y]=$ $C_{1}[D[Y]]$

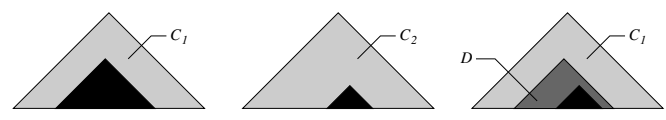


we have $t=C_{1}\left[\tau l_{1}\right]=C_{1}\left[D\left[\tau l_{2}\right]\right], u=C_{1}\left[\tau r_{1}\right]$, and $v=C_{1}\left[D\left[\tau r_{2}\right]\right]$. As $C_{1}\left[\tau l_{1}\right]=C_{1}\left[D\left[\tau l_{2}\right]\right]$, we have $\tau l_{1}=D\left[\tau l_{2}\right]$. Therefore, by Proposition 3.4, either there exist a variable $x$ and contexts $E_{1}[X]$ and $E_{2}[Y]$, such that $l_{1}=E_{1}[x], \tau x=E_{2}\left[\tau l_{2}\right]$, and $D[Y]=\left(\tau E_{1}\right)\left[E_{2}[Y]\right]$, or there exist a context $E[Y]$ and a term $l_{1}^{\prime}$ such that $l_{1}=E\left[l_{1}^{\prime}\right]$ and $D[Y]=(\tau E)[Y]$. We consider these two cases.

- If $l_{1}=E_{1}[x], \tau x=E_{2}\left[\tau l_{2}\right]$, and $D[Y]=$ $\left(\tau E_{1}\right)\left[E_{2}[Y]\right]$, then we let $\tau^{\prime}=\tau_{\mid \mathcal{V} \backslash\{x\}}$, and we have $\tau=\left(\tau^{\prime}, E_{2}\left[\tau l_{2}\right] / x\right)$. The term $l_{1}$ is linear and $x$ does not occur in $E_{1}$, thus $\tau E_{1}=\tau^{\prime} E_{1}$. Let $w=C_{1}\left[\left(\tau^{\prime}, E_{2}\left[\tau r_{2}\right] / x\right) r_{1}\right]$. We have

$$
\begin{gathered}
u=C_{1}\left[\tau r_{1}\right]=C_{1}\left[\left(\tau^{\prime}, E_{2}\left[\tau l_{2}\right] / x\right) r_{1}\right] \\
\longrightarrow{ }_{-}^{*} C_{1}\left[\left(\tau^{\prime}, E_{2}\left[\tau r_{2}\right] / x\right) r_{1}\right]=w
\end{gathered}
$$

and

$$
\begin{gathered}
v=C_{1}\left[D\left[\tau r_{2}\right]\right]=C_{1}\left[\left(\tau E_{1}\right)\left[E_{2}\left[\tau r_{2}\right]\right]\right] \\
=C_{1}\left[\left(\tau^{\prime} E_{1}\right)\left[E_{2}\left[\tau r_{2}\right]\right]\right] \\
=C_{1}\left[\left(\tau^{\prime}, E_{2}\left[\tau r_{2}\right] / x\right)\left(E_{1}[x]\right)\right] \\
=C_{1}\left[\left(\tau^{\prime}, E_{2}\left[\tau r_{2}\right] / x\right) l_{1}\right] \\
\longrightarrow C_{+}\left[\left(\tau^{\prime}, E_{2}\left[\tau r_{2}\right] / x\right) r_{1}\right]=w
\end{gathered}
$$

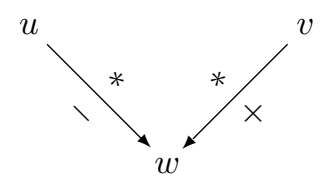

- If $l_{1}=E\left[l_{1}^{\prime}\right]$ and $D[Y]=(\tau E)[Y]$, then $\tau l_{1}=(\tau E)\left[\tau l_{1}^{\prime}\right]=D\left[\tau l_{1}^{\prime}\right]$. As we have $\tau l_{1}=D\left[\tau l_{2}\right]$, we get $D\left[\tau l_{1}^{\prime}\right]=D\left[\tau l_{2}\right]$, thus $\tau l_{1}^{\prime}=\tau l_{2}$. Let $\sigma$ be the most general unifier of $l_{1}^{\prime}$ and $l_{2}$ and $\eta$ such that $\tau=\eta \circ \sigma$. We have $u=C_{1}\left[\tau r_{1}\right]=C_{1}\left[\eta \sigma r_{1}\right]$ and $v=$ $C_{1}\left[(\eta \sigma E)\left[\eta \sigma r_{2}\right]\right]=C_{1}\left[\eta\left((\sigma E)\left[\sigma r_{2}\right]\right)\right] . \quad \mathrm{We}$ know that the critical pair $\left\langle\sigma r_{1},(\sigma E)\left[\sigma r_{2}\right]\right\rangle$ closes on a term, say $w_{0}$. Let $w=C_{1}\left[\eta w_{0}\right]$. We have

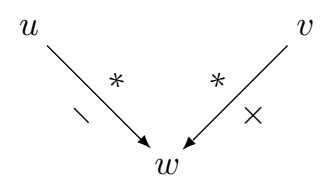

- The third case is similar to the second.

Definition 3.5 (Polarized Knuth-Bendix method). Let $\mathcal{P}$ be a left-linear finite polarized rewrite system and $\prec a$ reduction order, such that $l \succ r$ for each rule $l \longrightarrow$ or or $l \longrightarrow+r$ of $\mathcal{P}$.

While there is a non-joinable critical pair

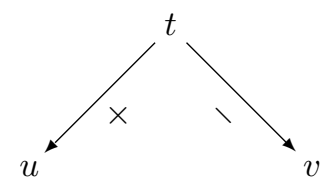

if $u \succ v$ and $u \longrightarrow_{-} v$ is a left-linear rewrite rule, add this rule to close the critical pair, if $v \succ u$ and $v \longrightarrow_{+} u$ is a left-linear rewrite rule, add this rule to close the critical pair, otherwise fail.

Proposition 3.6. Let $\mathcal{P}$ be a left-linear finite polarized rewrite system and $\prec$ a reduction order, such that $l \succ r$ for each rule $l \longrightarrow_{-}$or $l \longrightarrow_{+} r$ of $\mathcal{P}$. If the polarized Knuth-Bendix method applied to $\mathcal{P}$ succeeds, then reachability in $\mathcal{P}$ is decidable.

Proof. Let $\mathcal{P}^{\prime}$ be the left-linear finite polarized rewrite system built by the polarized Knuth-Bendix method. The rules of $\mathcal{P}^{\prime}$ are all derivable in $\mathcal{P}$, thus a proposition $t \longrightarrow{ }^{*} u$ has a proof in $\mathcal{P}$ if and only if it has a proof in $\mathcal{P}^{\prime}$.

As all the critical pairs of $\mathcal{P}^{\prime}$ are joinable, by Proposition 3.5, $\mathcal{P}^{\prime}$ is locally confluent. By construction, $l \succ r$ for each rule $l \longrightarrow_{-} r$ or $l \longrightarrow_{+}$ $r$ of $\mathcal{P}^{\prime}$. Thus, by Proposition 2.2, $\mathcal{P}^{\prime}$ terminates. By Proposition 3.2, as $\mathcal{P}^{\prime}$ is locally confluent and terminating, it has the cut-elimination property.

Thus, a proposition $t \longrightarrow^{*} u$ has a proof in $\mathcal{P}$ if and only if it has a proof in $\mathcal{P}^{\prime}$ if and only if it has a cutfree proof in $\mathcal{P}^{\prime}$. And, by Proposition 3.1, the existence of a cut-free proof for a proposition $t \longrightarrow^{*} u$ in $\mathcal{P}^{\prime}$ is decidable.

ExAMPLE 3 . Let $\mathcal{P}$ be the system defined in Example 1 and $\prec$ be the Knuth-Bendix order [13] with an equal weight 1 for all symbols and any precedence. For all rules $l \longrightarrow_{-} r$ or $l \longrightarrow_{+} r$ of $\mathcal{P}$, we have $l \succ r$. The only non-joinable critical pair is

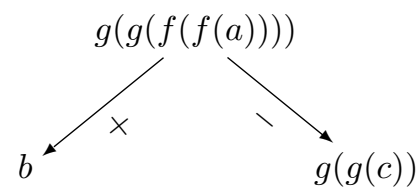

and it closes with the rule

$$
g(g(c)) \longrightarrow+b
$$

Let $\mathcal{P}^{\prime}$ be the system obtained by adding this rule to $\mathcal{P}$. The proposition $f(g(a)) \longrightarrow{ }^{*} g(g(h(a)))$ has the proof in $\mathcal{P}^{\prime}$

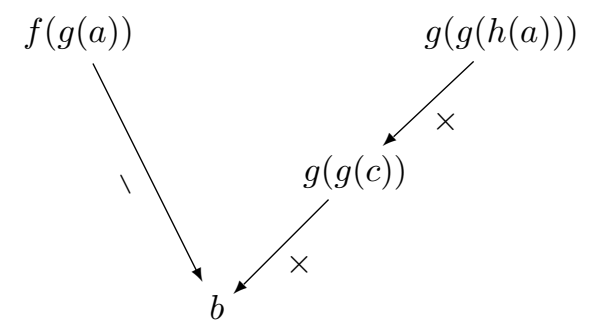

ThEOREM 3.1. Let $\mathcal{R}$ be a (non-polarized) finite rewrite system, $\mathcal{P}$ be a polarization of $\mathcal{R}$ and $\prec$ be a reduction order. If $\mathcal{P}$ is a left-linear polarized rewrite system, for all rules $l \longrightarrow_{-} r$ or $l \longrightarrow_{+} r$ of $\mathcal{P}, l \succ r$, and the polarized Knuth-Bendix method applied to $\mathcal{P}$ succeeds, then reachability in $\mathcal{R}$ is decidable.

Proof. From Propositions 2.1 and 3.6. 


\section{GROUND FINITE REWRITE SYSTEMS}

A ground rewrite system is a rewrite system such that for all rules $l \longrightarrow r$, both terms $l$ and $r$ are ground.

D. Lankford $[14,4]$ has observed that if $\prec$ is the Knuth-Bendix order with an equal weight 1 for all symbols and any precedence, $\mathcal{R}$ is a finite ground rewrite system, $\mathcal{R}^{\prime}$ is the equivalent system obtained by removing the rules of the form $l \longrightarrow l$ and reversing the rules $l \longrightarrow r$ such that $l \prec r$ into $r \longrightarrow l$, then the Knuth-Bendix method always succeeds on $\mathcal{R}^{\prime}$, and therefore equivalence in $\mathcal{R}$ is decidable.

We now want to prove that, in a similar way, reachability in a finite ground rewrite system is decidable [3].

Theorem 4.1 (Dauchet-Tison). Let $\mathcal{R}$ be a finite ground rewrite system. Then, the existence of a proof in $\mathcal{R}$ of a proposition $t{ }^{*} u$ is decidable.

Proof. Let $\prec$ be the Knuth-Bendix order with an equal weight 1 for all symbols and any precedence. This order is a reduction order and it is total on ground terms.

Without loss of generality, we can assume that $\mathcal{R}$ does not contain trivial rules of the form $l \longrightarrow l$. Let $\mathcal{P}$ be the polarization of $\mathcal{R}$ obtained by transforming each rule $l \longrightarrow r$ of $\mathcal{R}$ such that $l \succ r$, into a negative rule $l \longrightarrow \_r$, and reversing each rule $l \longrightarrow r$ such that $l \prec r$ into a positive rule $r \longrightarrow_{+} l$. By construction, $l \succ r$ for each rule $l \longrightarrow_{-} r$ or $l \longrightarrow_{+} r$ of $\mathcal{P}$.

Let $T$ be the finite set containing the left-hand sides of the rules of $\mathcal{P}$ and $T^{\prime}$ be the set of ground terms $t$ such that there exists a term $u$ in $T$ such that $t \prec u$ or $t=u$. As, for the Knuth-Bendix order, if $u$ is a ground term, the set of ground terms $t$ such that $t \prec u$ is always finite, the set $T^{\prime}$ is finite.

Then, the polarized Knuth-Bendix method applied to $\mathcal{P}$ generates rules whose left-hand sides and right-hand sides are in $T^{\prime}$. As there is only a finite number of such rules, the polarized Knuth-Bendix method applied to $\mathcal{P}$ terminates successfully.

Note that the original proof based on the construction of automata recognizing left-hand sides and right-hand sides of rules [3] also uses implicitly the idea of reversing rewrite rules. For instance, with the rule $f(a) \longrightarrow g(b)$, it builds an automaton recognizing $f(a)$ in $s$

$$
\begin{gathered}
a \longrightarrow s_{1} \\
f\left(s_{1}\right) \longrightarrow s
\end{gathered}
$$

another recognizing $g(b)$ in $s^{\prime}$

$$
\begin{gathered}
b \longrightarrow s_{1}^{\prime} \\
g\left(s_{1}^{\prime}\right) \longrightarrow s^{\prime}
\end{gathered}
$$

and takes the rewrite rule
This construction can be decomposed in two steps, one transforming the rule $f(a) \longrightarrow g(b)$ into the rewrite system

$$
\begin{gathered}
a \longrightarrow s_{1} \\
f\left(s_{1}\right) \longrightarrow s \\
s \longrightarrow s^{\prime} \\
s_{1}^{\prime} \longrightarrow b \\
s^{\prime} \longrightarrow g\left(s_{1}^{\prime}\right)
\end{gathered}
$$

where $f(a) \longrightarrow f\left(s_{1}\right) \longrightarrow s \longrightarrow s^{\prime} \longrightarrow g\left(s_{1}^{\prime}\right) \longrightarrow g(b)$ and then reversing the two rules

$$
\begin{gathered}
s_{1}^{\prime} \longrightarrow b \\
s^{\prime} \longrightarrow g\left(s_{1}^{\prime}\right)
\end{gathered}
$$

The first step is in fact not needed.

\section{PUSHDOWN SYSTEMS}

As another corollary of our result, we also get the decidability of reachability for pushdown systems [1].

Definition 5.1 (Pushdown system). Consider a language containing a set $S$ of unary function symbols called stack symbols, a set $Q$ of unary function symbols called states and a constant $\varepsilon$. A pushdown system is a finite rewrite system with rules of the form: pop rules

$$
p(\gamma(x)) \longrightarrow q(x)
$$

where $\gamma$ is a stack symbol and $p$ and $q$ are states, push rules

$$
p(x) \longrightarrow q(\gamma(x))
$$

where $\gamma$ is a stack symbol and $p$ and $q$ are states, and neutral rules

$$
p(x) \longrightarrow q(x)
$$

where $p$ and $q$ are states.

Theorem 5.1 (Bouajjani-Esparza-Maler). Let $\mathcal{R}$ be a pushdown system. Then, the existence of a proof in $\mathcal{R}$ of a proposition $t \longrightarrow^{*} u$ is decidable.

Proof. Consider a total precedence on function symbols such that stack symbols are larger than states and let $\prec$ be the lexicographic path order [12] relative to this precedence. The order $\prec$ is a reduction order and $p(\gamma(x)) \succ q(x)$ for all $\gamma, p$ and $q$.

Pop rules are polarized as

$$
p(\gamma(x)) \longrightarrow_{-} q(x)
$$

push rules as

$$
q(\gamma(x)) \longrightarrow_{+} p(x)
$$

and neutral rules according to the precedence.

Critical pairs have the form

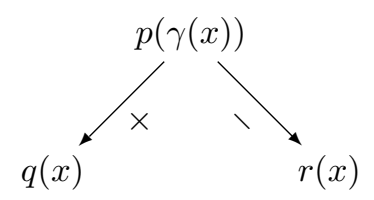



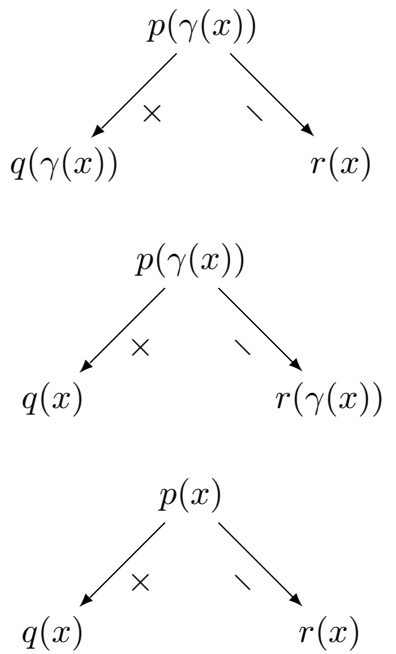

all these critical pairs are closed by adding a pop rule, a push rule, or a neutral rule. As there are a finite number of such rules, the polarized Knuth-Bendix method always terminates successfully.

Thus reachability in pushdown systems is decidable.

Note that we get, in this way, the decidability of reachability in pushdown systems, but not of alternating pushdown systems [1], that requires moving from polarized rewrite systems to polarized sequent calculus modulo [2].

\section{ACKNOWLEDGEMENTS}

The authors want to thank Nao Hirokawa, Gérard Huet, Jean-Pierre Jouannaud, Claude Kirchner, and Vincent van Oostrom, for useful discussions on this paper and bibliographical indications. This work is supported by the ANR-NSFC project LOCALI (NSFC 61161130530 and ANR 11 IS02 002 01) and the Chinese National Basic Research Program (973) Grant No. 2014 CB340302.

\section{REFERENCES}

[1] A. Bouajjani, J. Esparza, and O. Maler. Reachability analysis of pushdown automata: Application to modelchecking. In A. W. Mazurkiewicz and J. Winkowski, editors, Concurrency theory, volume 1243 of Lecture Notes in Computer Science, pages 135-150, 1997.

[2] G. Burel, G. Dowek, and Y. Jiang. Automata, resolution and cut-elimination. manuscript, 2015.

[3] M. Dauchet and S. Tison. Decidability of confluence for ground term rewriting systems. In Fundamentals of Computation Theory, pages 80-89, 1985.

[4] N. Dershowitz. Completion and its applications. In H. Aït-Kaci and M. Nivat, editors, Resolution of Equations in Algebraic Structures, volume 2, chapter 2, pages 31-86. Academic Press, 1989.

[5] N. Dershowitz and Z. Manna. Proving termination with multiset orderings. Communications of the ACM, 22(8):465-476, 1979.
[6] G. Dowek. What is a theory? In H. Alt and A. Ferreira, editors, Theoretical Aspects of Computer Science, volume 2285 of Lecture Notes in Computer Science, pages 50-64, 2002.

[7] G. Dowek. Confluence as a cut elimination property. In R. Nieuwenhuis, editor, Rewriting Technique and Applications, volume 2706 of Lecture Notes in Computer Science, pages 2-13, 2003.

[8] G. Dowek. Polarized resolution modulo. In C.S. Calude and V. Sassone, editors, Theoretical Computer Science, volume 323 of IFIP Advances in Information and Communication Technology, pages 182-196. Springer, 2010.

[9] N. Hirokawa. Commutation and signature extension. In A. Tiwari and T. Aoto, editors, International Workshop on Confluence, 2015.

[10] G. Huet. Confluent reductions: abstract properties and applications to term rewriting systems. Journal of the Association of Computing Machinery, 27(4):797-821, 1980.

[11] J.-P. Jouannaud and H. Kirchner. Completion of a set of rules modulo a set of equations. SIAM Journal of Computing, 15(4):1155-1194, 1986.

[12] S. Kamin and J.-J. Lévy. Attempts for generalizing the recursive path ordering. Unpublished manuscript.

[13] D. Knuth and P. Bendix. Simple word problems in universal algebras. In Computational Problems in Abstract Algebra, pages 263-297. Pergamon, 1970.

[14] D. S. Lankford. Canonical inference. Technical report, Louisiana Tech. University, 1975. 